6. Cyster JG, Ansel KM, Reif K, et al. Follicular stromal cells and lymphocyte homing to follicles. Immunol Rev $2000 ; 176$ : 181-93.

7. Kaldjian EP, Gretz JE, Anderson A0, et al. Spatial and molecular organization of lymph node T cell cortex: a labyrinthine cavity bounded by an epithelium-like monolayer of fibroblastic reticular cells anchored to basement membrane-like extracellular matrix. Int Immunol 2001; 13: 1243-53.

8. Anderson AO, Shaw S. T cell adhesion to endothelium: the FRC conduit system and other anatomic and molecular features which facilitate the adhesion cascade in lymph node. Semin Immunol 1993; 5 : 271-82.
9. Gunn MD, Ngo VN, Ansel KM, et al. A B-cell-homing chemokine made in lymphoid follicles activates Burkitt's lymphoma receptor-1. Nature 1998 ; 391 : 799-803.

10. Hase H, Kanno Y, Kojima M, et al. BAFF/BLyS can potentiate $B$-cell selection with the $B$-cell coreceptor complex. Blood 2004 ; 103 : 2257-65.

\title{
NOUVELLE
}

\section{L'angiogenèse passe sous contrôle du couple Delta-Notch}

Steven Suchting, Catarina Freitas, Anne Eichmann
Inserm U833, Collège de France,

11, place Marcelin Berthelot, 75005 Paris, France.

anne.eichmann@college-de-France.fr
> Pendant le développement embryonnaire, la croissance des vaisseaux sanguins, ou angiogenèse, s'opère suivant un contrôle précis. Insuffisante, ou au contraire excessive, l'angiogenèse per-
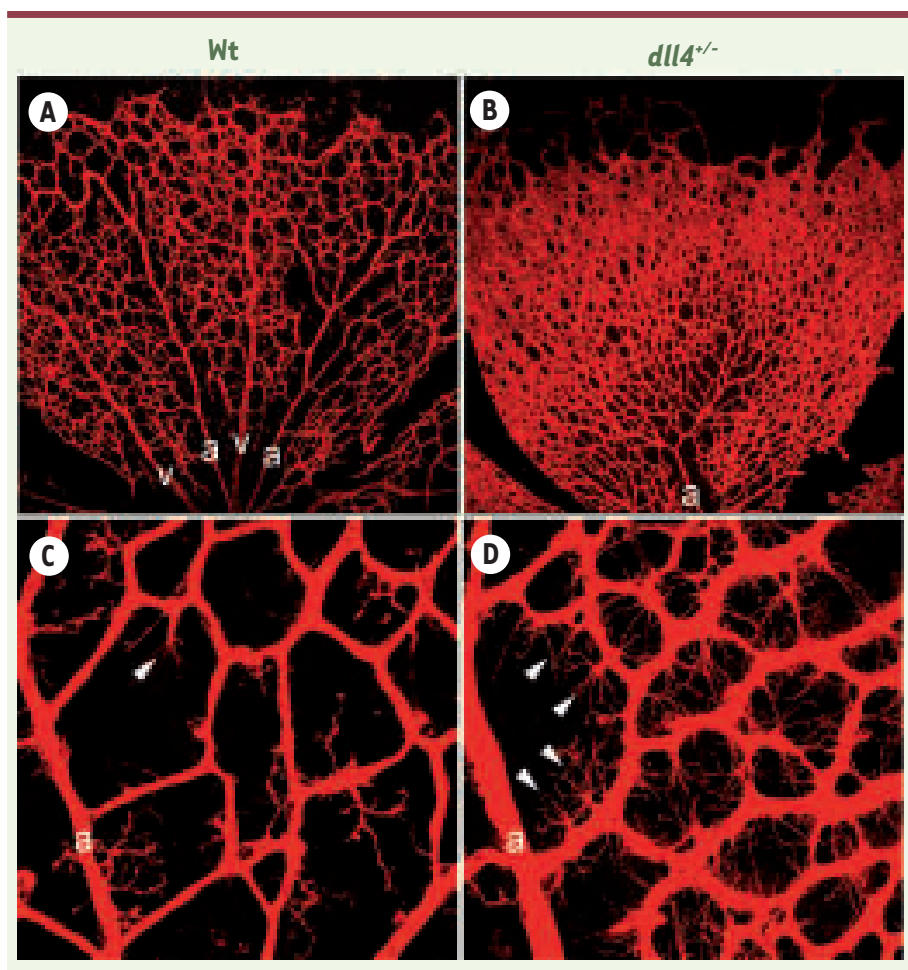

Figure 1. Augmentation du branchement des vaisseaux de la rétine et de la formation de tip cells après délétion d'un allèle dll4. A. Réseau vasculaire normal avec alternance d'artères et de veines connectées par des capillaires. B. Les vaisseaux dll $4^{+/-}$montrent un branchement excessif et une fusion excessive du réseau vasculaire. À plus fort grossissement (C, D) les vaisseaux $d l l 4^{+/-}$(D) présentent plus de tip cells, chacune étant à l'origine d'un nouveau point de branchement. $a$ : artères ; $v$ : veines; les têtes de flèches montrent quelques tip cells dans chaque image. turbera la circulation et souvent entrầnera la mort des embryons.

Parmi les molécules régulatrices de l'angiogenèse, la mieux étudiée est un facteur de croissance pro-angiogénique, le VEGF

(vascular end othelial growth factor). Le VEGF est fortement exprimé par les tissus en hypoxie, qui recrutent de nouveaux vaisseaux à partir des territoires environnants pour assurer leurs besoins en oxygène et nutriments. Plus la vascularisation des tissus, et donc leur oxygénation, se développe, plus l'expression du
VદGF diminue. Cet élégant mécanisme de rétrocontrôle négatif est une clef de la régulation de la croissance des vaisseaux. Toutefois, les vaisseaux exposés à de fortes concentrations de VEGF ne bourgeonneront pas tous. La réponse vasculaire au VEGF est affinée par d'autres mécanismes régulateurs. Sept articles récemment publiés dans Nature, Development et Proc Natl Acad Sci USA révèlent le rôle d'un autre partenaire, Delta-like 4 (DII4), un ligand de Notch, dans la restriction de la réponse des vaisseaux au VEGF [1-7].

La famille des récepteurs transmembranaires Notch et celles de leurs ligands jouent un rôle essentiel dans les phénomènes de spécification et de différenciation, pour de multiples types cellulaires, lors du développement embryonnaire [8, 9]. Parmi les molécules intervenant dans la voie Notch impliquées dans le développement vasculaire, le ligand DII4 a suscité un intérêt particulier puisqu'il est spécifique des artères et capillaires en bourgeonnement. Les sept nouvelles études, dont la nôtre, ont examiné la contribution de DII4/Notch à l'angiogenèse embryonnaire et pathologique. Les divers modèles expérimentaux utilisés démontrent que l'absence de la signalisation Dll4/Notch, soit par ablation génétique, inhibition pharmacologique, ou administration d'anticorps antagonistes, provoque une angiogenèse excessive par bourgeonnement vasculaire. Notre étude, 
par exemple, montre que l'ablation d'un seul allèle dll4 chez la souris accroît de façon spectaculaire le bourgeonnement et le branchement vasculaires au sein de la rétine (Figure 1).

Par quels mécanismes cellulaires et moléculaires naît cette profusion de vaisseaux? La microscopie à haute résolution a permis d'observer un grand nombre de tip cells endothéliales un peu partout dans le plexus vasculaire des rétines $d l l 4^{+/-}$. Les tip cells sont situées à l'extrémité des capillaires en bourgeonnement. Ce sont des cellules dotées de nombreuses extensions filopodiales qui fonctionnent comme des senseurs de l'environnement et guident la croissance des vaisseaux. Cette augmentation significative des tip cells chez les animaux $d l l 4^{+/-}$indique que, dans les conditions normales, leur formation est inhibée par une signalisation impliquant DII4. En bloquant avec un inhibiteur pharmacologique spécifique la voie de signalisation Notch activée par la fixation de DII4 à son récepteur, nous avons par ailleurs observé un phénotype similaire à celui du mutant $d l l 4^{+/-}$, ce qui montre que Notch est l'intermédiaire par lequel DII4 agit dans ces vaisseaux.

Comment une déficience dans la signalisation de la voie Notch peut-elle entraîner une profusion vasculaire? Le VEGF est, comme nous l'avons vu précédemment, nécessaire au développement des vaisseaux dans la rétine. Nous avons pensé que la réponse



Figure 2. Modèle proposé pour le mécanisme d'action de Dll4/Notch. En situation normale (A), le VEGF provoque l'acquisition par une cellule endothéliale (CE) d'un phénotype de tip cell ce qui se traduit par l'émergence d'un bourgeonnement capillaire. Le VEGF induit également l'expression de dll4. En se liant à son récepteur Notch présent sur la CE adjacente, DII4 inhibera la formation d'une autre tip cell en induisant une altération du taux d'expression des récepteurs au VEGF, rendant la cellule moins sensible au VEGF. Les vaisseaux $d l l 4^{+/-}$(B) sont incapables de transmettre le signal inhibiteur et le nombre de CE qui répondent de façon inapproprié au VEGF et deviennent des tip cells augmente. II en résulte un branchement et une fusion excessifs de ces vaisseaux. ment de ces angiogenèses pathologiques. Des anticorps bloquant la signalisation DII4 ont ainsi été développés, indépendamment, par deux sociétés de biotechnologies américaines $[6,7]$. Dans des modèles de xénogreffes tumorales humaines chez la souris, l'administration d'anticorps anti-Dll4 provoque une augmentation spectaculaire de l'angiogenèse tumorale avec, de manière paradoxale en apparence, une réduction de la taille des tumeurs. Les nombreux nouveaux vaisseaux sont en fait hérissés de tip cells, et deviennent non fonctionnels, incapables d'assurer la circulation sanguine et la croissance tumorale qui en dépend. II faut souligner que ce blocage de Dll4 s'est avéré efficace dans le traitement de tumeurs résistantes à des thérapies anti-VEGF. Avec la mise au point d'anticorps humanisés anti-DII4, les thérapies anti-angiogéniques pourraient donc prochainement s'enrichir d'un nouvel outil élargissant leur spectre d'application. $\diamond$

Angiogenesis under

Delta-Notch couple control

\section{RéFÉRENCES}

1. Suchting S, Freitas C, le Noble F, et al. The Notch ligand Delta-like 4 negatively regulates endothelial tip cell formation and vessel branching. Proc Natl Acad Sci USA 200712 février online.

2. Siekmann AF, Lawson ND. Notch signalling limits angiogenic cell behaviour in developing zebrafish arteries. Nature 2007 ; 445 : 781-4.

3. Lobov IB, Renard RA, Papadopoulos N, et al. Deltalike ligand 4 (DII4) is induced by VEGF as a negative regulator of angiogenic sprouting. Proc Natl Acad Sci USA 200712 février online.

4. Leslie JD, Ariza-McNaughton L, Bermange AL, et al. Endothelial signalling by the Notch ligand Delta-like 4 restricts angiogenesis. Development $2007 ; 134$ : 839-44.

5. Hellstrom M, Phng LK, Hofmann JJ, et al. DII4 signalling through Notchl regulates formation of tip cells during angiogenesis. Nature 2007 ; $445: 776-80$.

6. Ridgway J, Zhang G, Wu Y, et al. Inhibition of DII4 signalling inhibits tumour growth by deregulating angiogenesis. Nature 2006 ; 444 : 1083-7.

7. Noguera-Troise I, Daly C, Papadopoulos NJ, et al. Blockade of DII4 inhibits tumour growth by promoting non-productive angiogenesis. Nature $2006 ; 444: 1032-7$

8. Cormier S, Souilhol C, Babinet C, Cohen-Tannoudji M. Notch signalling pathway and early mammalian embryogenesis. Med Sci (Paris) 2007 ; $23: 26-8$.

9. Six $\varepsilon$, Andre Schmutz I, Cavazzana-Calvo M. Notch ligands Delta and lymphoid development niches. Med Sci (Paris) 2007 ; $23: 21-4$ 